Gut, 1988, 29, 1286-1288

Methods

\title{
Markers for faecal fat estimation in monitoring steatorrhoea in cystic fibrosis
}

\author{
J GilberT, J KELLEHER, M P WALTERS, AND J M LiTTLEWOOD \\ From the Regional Cystic Fibrosis Unit and Department of Medicine, St James's University Hospital, Leeds
}

SUMmaRY Polyethylene glycol (PEG) 4000 is one of numerous substances used as non-absorbable markers to correct for variable faecal output when assessing daily faecal losses of nutrients. The introduction of enteric coated micro-encapsulated pancreatic enzyme (EMPE) preparations has greatly improved the control of fat malabsorption in cystic fibrosis and chronic pancreatitis patients. Unfortunately, these enzyme preparations contain significant quantities of PEG 4000 or polyvinyl pyrolidine (PVP) as components of the enteric coating and thus PEG 4000 cannot be used either as a faecal marker, or in intubation studies, if these enzyme preparations are being used.

Minimising faecal fat output is essential in the management of cystic fibrosis (CF) if the benefits of the high energy, normal fat diet which is now recommended are to be fully realised. Optimal fat balance can only be achieved if faecal fat output is monitored ${ }^{1}$ and continuous faecal markers should be used if accurate assessment of faecal fat output is to be obtained. ${ }^{2}$ In the past many different substances have been used for this purpose. ${ }^{3}$ Polyethylene glycol 4000 is widely used and has been shown to give accurate results for diagnostic purposes in patients not yet receiving treatment. ${ }^{4}$ The use of radio-opaque pellets (ROP) has also been advocated on the grounds of simplicity, safety and minimal faecal handling in the laboratory. ${ }^{45}$

It is known that certain oral pharmaceutical preparations contain PEG, and inconsistent results using PEG as a marker in CF patients on a variety of medications lead us to reconsider its value for routine use in this group. We have compared PEG and ROP as continuous faecal markers in CF patients taking EMPE preparations, in doses adjusted according to the fat content of the meal in order to minimise symptoms and faecal fat excretion.

Address for correspondence: Dr J Kelleher, Dept of Medicine, St James's University Hospital, Leeds LS9 7TF.

Received for publication 7 April 1988.
Methods

PATIENTS

Radio-opaque pellets were prepared by cutting 1.0 $1.5 \mathrm{~mm}$ lengths of Portex $2.0 \mathrm{~mm}$ internal diameter radio-opaque tubing; these were extracted overnight with toluene to remove the plasticiser and, after drying, were packaged in gelatin capsules, each of which contained eight pellets. For seven days $17 \mathrm{CF}$ patients received $500 \mathrm{mg}$ PEG (Sandoz Products Ltd, London) and eight ROP three times daily, and 11 received only eight ROP three times per day. Normal diet and medication were continued throughout the seven days. During the last 48 hours all stools were collected in separate strong polyethylene bags, which fitted directly on to a commode.

Stools were frozen and then $x$-rayed. Radioopaque pellets were easily distinguished on $x$-ray films and were counted. The pooled two day collections were then homogenised and analysis was performed for PEG by the method of Malawer ${ }^{b}$ and for fat by the method of van de Kamer et al. ${ }^{7}$

Daily faecal fat output was calculated using each marker.

\section{Results}

In the CF patients studied, daily faecal fat output ranged from $34.7 \mathrm{mmol}$ to $274 \mathrm{mmol}$ (mean 100.4 


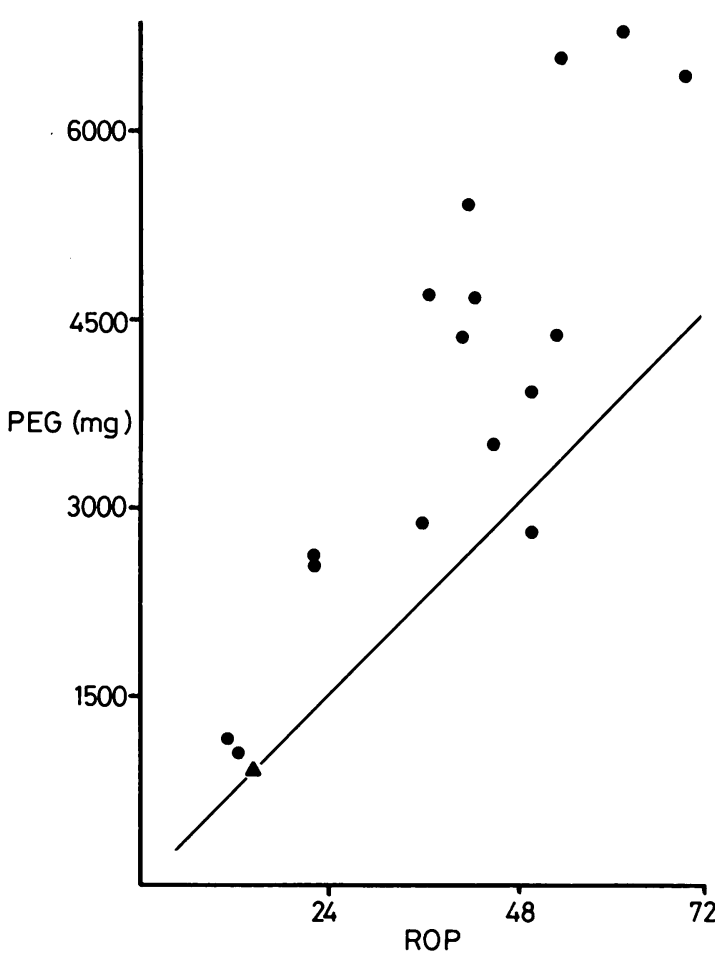

Figure Comparison of total PEG like material and ROP in two day stool collections. $\triangle$ represents the patient who was not taking any pancreatic enzyme supplements.

mmol) when using ROP as the marker, and from $13 \cdot 6$ mmol to $162.4 \mathrm{mmol}$ (mean $63.2 \mathrm{mmol}$ ) when using PEG.

The correlation between PEG and ROP content in the 48 hour faecal collections is shown in the Figure, and it can be seen that despite the close correlation $(r=0 \cdot 87)$, in all except one of the patients taking pancreatic supplements, there is proportionately more PEG than ROP present. Use of these marker concentrations to calculate daily faecal output results in an error of up to $52 \%$ (mean $35 \%$ ) when PEG was the marker. In the one patient not taking pancreatic supplements, there was only a $6 \%$ difference in the results obtained using the two markers.

Two day faecal collections from $11 \mathrm{CF}$ patients taking only ROP as the continuous marker were also analysed for PEG, and contained between $61 \mathrm{mg}$ and $2815 \mathrm{mg}$ of PEG.

The quantity of PEG (or PEG reacting substances) was estimated in a range of medications which are frequently taken by $\mathrm{CF}$ patients. These analyses were undertaken on untreated medications and also after incubation with a control faecal homogenate for six hours at $37^{\circ} \mathrm{C}$. As seen in the Table, significant
Table PEG analysis of medications

\begin{tabular}{llcc}
\hline & \multicolumn{3}{l}{ Known PEG PEG analysis PEG analysis } \\
Medication & content & with faeces & without faeces \\
\hline Amoxycillin cap & $\mathrm{Nil}$ & $\mathrm{Nil}$ & $\mathrm{Nil}$ \\
Amoxycillin liquid & $\mathrm{Nil}$ & $\mathrm{Nil}$ & $\mathrm{Nil}$ \\
Ciprofloxacin tab & $1 \cdot 3$ & $5 \cdot 0$ & $3 \cdot 2$ \\
Cotazyme cap & $\mathrm{Nil}$ & $5 \cdot 4$ & $2 \cdot 3$ \\
Cotrimoxazole tab & $\mathrm{Nil}$ & $\mathrm{Nil}$ & $\mathrm{Nil}$ \\
Cotrimoxazole liquid & $\mathrm{Nil}$ & $3 \cdot 0$ & $2 \cdot 0$ \\
Creon cap & $75 \cdot 0$ & $72 \cdot 0$ & $68 \cdot 0$ \\
Erythromycin 500 mg & $7 \cdot 1$ & $16 \cdot 0$ & $13 \cdot 0$ \\
$\quad$ tab & & & \\
Flucloxacillin cap & $\mathrm{Nil}$ & $\mathrm{Nil}$ & $\mathrm{Nil}$ \\
Flucloxacillin liquid & $\mathrm{Nil}$ & $\mathrm{Nil}$ & $\mathrm{Nil}$ \\
Nutrixyme tab & $\mathrm{Nil}$ & $5 \cdot 6$ & $4 \cdot 0$ \\
Pancrease cap* & $\mathrm{Nil}$ & $60 \cdot 0$ & $56 \cdot 0$ \\
Pancrex V cap & $\mathrm{Nil}$ & $2 \cdot 3$ & $0 \cdot 9$ \\
Pancrex V Forte tab & $\mathrm{Nil}$ & $14 \cdot 0$ & $10 \cdot 0$ \\
Salbutamol tab & $\mathrm{Nil}$ & $\mathrm{Nil}$ & $\mathrm{Nil}$ \\
Salbutamol liq & $\mathrm{Nil}$ & $6 \cdot 5$ & $9 \cdot 5$ \\
Sodium fusidate tab & $\mathrm{Nil}$ & $43 \cdot 0$ & $63 \cdot 0$ \\
Vitamin A \& D cap & $\mathrm{Nil}$ & $\mathrm{Nil}$ & $\mathrm{Nil}$ \\
Vitamin A \& D liquid & $\mathrm{Nil}$ & $105 \cdot 0$ & $240 \cdot 0$ \\
Vitamin E tab & $\mathrm{Nil}$ & $\mathrm{Nil}$ & $\mathrm{Nil}$ \\
Vitamin E liquid & 400 & $192 \cdot 0$ & $372 \cdot 0$ \\
& & & \\
\hline
\end{tabular}

All amounts expressed as $\mathrm{mg} / 5 \mathrm{ml}$, capsule or tablet. *contains PVP.

quantities of PEG like material were present in many of these preparations. Of particular importance is the large quantity present in each capsule of both Creon (Duphar Laboratories Ltd, Southampton) and Pancrease (Ortho-Cilag Pharmaceutical Ltd, Bucks). Incubation in vitro with a faecal suspension had little effect on the PEG content of Creon or Pancrease, but did reduce considerably the apparent PEG content of the vitamin E suspension and sodium fusidate tablets. The agreement between stated and assayed PEG content of Creon and vitamin $E$ liquid is close in the absence of added faeces. The reduction in apparent PEG content when some of these preparations are incubated with faeces is unexplained.

\section{Discussion}

Some medications, such as EMPE, which are frequently taken by CF patients, are known to contain PEG or PVP (which will also be determined in the usual turbidimetric method used for PEG analysis). It is also evident that many other medications contain substances which will cause a positive result for PEG and these may be further altered when incubated in faeces. Both the EMPE preparations which are currently available in this country (Creon and Pancrease) contain relatively large quantities of PEG-reacting substances, and some of our CF patients are taking as many as 50 capsules, which 
represents up to $3600 \mathrm{mg}$ of PEG per day. These findings explain the discrepancy between the results obtained using PEG and ROP as markers and the finding of PEG reacting substances in the faeces of patients not taking any PEG as marker.

Polyethylene glycol may be an acceptable marker for diagnostic purposes but cannot be used to monitor treatment of patients taking pancreatic supplementation on certain other medications. Radio-opaque pellets give accurate results in this situation and are easier to estimate.

The range of drugs tested in this study is by no means exhaustive, but the frequency with which PEG like material is found in pharmaceutical preparations suggests that extreme caution should be taken in the interpretation of results where PEG 4000 is used as the continuous non-absorbable marker. The previously used pancreatic enzyme preparations, such as Cotazyme (Organon Laboratories Ltd, Cambridge) and Nutrizyme (Merck Ltd, Alton, Hants), contain much less PEG like material than the newer EMPE preparations.

Radio-opaque pellets have now been used in many thousands of balance studies and harmful side effects have not been described (Cummings $\mathrm{J} \mathrm{H}$, personal communication). Our present policy is to use ROP in all patients capable of swallowing a small capsule intact. In younger children, we use a three day collection without any markers.

We thank the Cystic Fibrosis Research Trust for funding Dr J Gilbert and Mr M P Walters.

\section{References}

1 Kelleher J. Laboratory measurement of nutrition in cystic fibrosis. J R Soc Med 1987; 80 [Suppl 15]: 25-9.

2 Walker BE, Kelleher J, Davies T, Losowsky MS. Chemical faecal fat using single stools. Scand J Gastroenterol 1971; 6: 277-80.

3 Morgan JB. Use of non-absorbable markers in studies of human nutrient absorption. Hum Nutr Appl Nutr 1986; 40A: 399-411.

4 Simpson FG, Hall GP, Kelleher J, Losowsky MS. Radioopaque pellets as faecal markers for faecal fat estimation in malabsorption. Gut 1979; 20: 581-4.

5 Cummings JH, Jenkins DJA, Wiggins HS. Measurements of the mean transit time of dietary residue through the human gut. Gut 1976; 17: 210-8.

6 Malawer SJ, Powell DW. An improved turbidimetric analysis of PEG using an emulsifier. Gastroenterology $1967 ; 53 ; 250-6$.

7 Kamer JH van de, Bokkel Huinink $H$ ten, Weyers HA. Rapid method of determination of fat in feces. $J$ Biol Chem 1949; 177: 347-55. 